\title{
SOME APPLICATIONS OF CONVOLUTION OF OPERATORS ON BANACH SPACES
}

\author{
PATRICK N. DOWLING
}

(Communicated by Paul S. Muhly)

\begin{abstract}
Using convolution between functions and cone absolutely summing operators, we obtain characterizations of Banach spaces with the RadonNikodym property, the analytic Radon-Nikodym property and Banach spaces not containing a copy of $c_{0}$.
\end{abstract}

\section{INTRODUCTION}

In [B2] Blasco gave characterizations of the Radon-Nikodym property and the analytic Radon-Nikodym property using convolution between functions and cone absolutely summing operators. The aim of this note is to improve Blasco's characterization of the analytic Radon-Nikodym property and also to extend Blasco's results to give a new characterization of Banach spaces not containing a copy of $c_{0}$. In fact, we will prove a general result that give the characterizations of the Radon-Nikodym property, the analytic Radon-Nikodym property, and Banach spaces not containing $c_{0}$ as easy corollaries.

\section{PReliminaries AND Definitions}

Throughout this note $G$ will denote a compact abelian metrizable group, $\mathscr{B}(G)$ denotes the $\sigma$-algebra of Borel subsets of $G$, and $\lambda$ is normalized Haar measure on $G$. The dual group of $G$ will be denoted by $\Gamma$ and is a countable discrete abelian group.

Definition 2.1. Let $1<p<\infty$. Given a bounded linear operator $T: L^{p}(G) \rightarrow$ $X$ and a function $g \in L^{1}(G)$, we define an operator $g * T: L^{p}(G) \longrightarrow X$ by $(g * T)(f)=T(f * g)$ for all $f \in L^{p}(G)$. Clearly, $\|g * T\| \leq\|g\|_{1}\|T\|$.

Definition 2.2. Let $1 \leq r<\infty, 1<p<\infty$, and $1 / p+1 / q=1$. A bounded linear operator $T: L^{p}(G) \rightarrow X$ is called a positive $r$-summing operator if there exists a constant $C$ such that for every $\left\{f_{i}\right\}_{i=1}^{n}$ in $L^{p}(G)$ with $f_{i} \geq 0$ for $i=1,2, \ldots, n$ the following inequality holds,

$$
\left(\sum_{i=1}^{n}\left\|T\left(f_{i}\right)\right\|^{r}\right)^{1 / r} \leq C \sup \left\{\left(\sum_{i=1}^{n}\left|\int_{G} f_{i}(t) g(t) d \lambda(t)\right|^{r}\right)^{1 / r}:\|g\|_{q}=1\right\} \text {. }
$$

Received by the editors December 7, 1990 and, in revised form, February 23, 1991. 1991 Mathematics Subject Classification. Primary 46B22. 
The positive $r$-summing norm of $T$ is given by the infinum of all such constants $C$ and is denoted by $\|T\|_{p, r}$. The space of positive $r$-summing operates from $L^{P}(G)$ to $X$ is denoted by $\Lambda_{r}\left(L^{p}(G), X\right)$. The space of operators we will be most interested in is $\Lambda_{1}\left(L^{p}(G), X\right)$, which is often referred to as the space of cone absolutely summing operators $[\mathrm{B} 1, \mathrm{~S}]$.

Definition 2.3. Let $1 \leq p<\infty$ and $1 / p+1 / q=1$. A bounded linear operator $T: L^{p}(G) \rightarrow X$ is called representable if there exists a function $f \in$ $L^{q}(G, X)$ such that $T(h)=\int_{G} f(t) h(t) d \lambda(t)$ for all $h \in L^{p}(G)$. In this case we will write $T=T_{f}$. It is easily seen that $\left\|T_{f}\right\| \leq\|f\|_{q}$.

Remark. Schaefer [S] proved that if $f \in L^{q}(G, X)$ then $T_{f} \in \Lambda_{1}\left(L^{p}(G), X\right)$ and $\left\|T_{f}\right\|_{p, 1}=\|f\|_{q}$. Also, it is easily seen that if $g \in L^{1}(G)$ then $g * T_{f}=T_{g * f}$ and $\left\|g * T_{f}\right\|_{p, 1} \leq\|g\|_{1}\left\|T_{f}\right\|_{p, 1}$. These observations have been extended by Blasco [B2].

Theorem 2.1 (Blasco). Let $1 \leq r<\infty, 1<p<\infty, 1 / p+1 / q=1$, and let $g$ be a positive element of $L^{1}(G)$.

(i) If $T \in \Lambda_{r}\left(L^{p}(G), X\right)$ then $g * T \in \Lambda_{r}\left(L^{p}(G), X\right)$ and $\|g * T\|_{p, r} \leq$ $\|g\|_{1}\|T\|_{p, r}$.

(ii) If $T: L^{p}(G) \rightarrow X$ is bounded and linear and $g \in L^{p}(G)$, then there is a continuous function $h: G \rightarrow X$ such that $g * T=T_{h}$. If, in addition, we also assume that $T \in \Lambda_{1}\left(L^{p}(G), X\right)$ then $\|h\|_{q} \leq\|g\|_{1}\|T\|_{p, 1}$.

Definition 2.4. A sequence of functions $\left\{g_{n}\right\}_{n=1}^{\infty}$ in $L^{1}(G)$ is called an approximate identity if

(a) $\int_{G} g_{n}(t) d \lambda(t)=1$ for all $n \in \mathbb{N}$,

(b) $\sup _{n} \int_{G}\left|g_{n}(t)\right| d \lambda(t)<\infty$, and

(c) $\lim _{n \rightarrow \infty} \int_{U} g_{n}(t) d \lambda(t)=1$ for all neighborhoods $U$ of 1 in $G$.

Remark. Since $G$ is a compact abelian metrizable group approximate identities exist. In fact, approximate identities of positive functions exist [R, p. 23].

\section{RADON-NIKODYM PROPERTIES AND APPLICATIONS}

As in the previous section, $G$ will denote a compact abelian metrizable group and $\Gamma$ will denote the dual group of $G$. Let $M$ be a subset of $\Gamma$ and let $X$ be a Banach space. Let us recall that for an $X$-valued measure, $\mu$, on $\mathscr{B}(G)$ we can define $\mathbb{E}(\mu \mid \pi)$ by

$$
\mathbb{E}(\mu \mid \pi)=\sum_{E \in \pi} \frac{\mu(E)}{\lambda(E)} \chi_{E},
$$

where $\pi$ is a finite measurable partition of $G$, along with the convention $\frac{0}{0}=0$. We say that $\mu$ is of bounded average range (respectively, of bounded variation) if $\sup _{\pi}\|\mathbb{E}(\mu \mid \pi)\|_{L^{\infty}(G ; X)}<\infty$ (respectively, $\left.\sup _{\pi}\|\mathbb{E}(\mu \mid \pi)\|_{L^{1}(G ; X)}<\infty\right)$, where the supremum is taken over all finite measurable partitions of $G$.

Definition 3.1. (a) $X$ is said to have type I- $M$-Radon-Nikodym property (type I- $M$-RNP) if every $X$-valued measure, $\mu$, on $\mathscr{B}(G)$ that is of bounded average range and such that $\hat{\mu}(\gamma)=0$ for all a $\gamma \notin M$ has Radon-Nikodym derivative with respect to $\lambda$. 
(b) $X$ is said to have type II- $M$-Radon-Nikodym property (type II- $M$-RNP) if every $X$-valued measure, $\mu$, on $\mathscr{B}(G)$ that is of bounded variation, which is absolutely continuous with respect to $\lambda$, and such that $\hat{\mu}(y)=0$ for all $\gamma \notin M$ has a Radon-Nikodym derivative with respect to $\lambda$.

Remarks. (i) Type I- $M$-RNP was introduced by Edgar [E]. More information on types I and II- $M$-RNP can be found in [D2].

(ii) If $G=\mathbb{T}$ then $\Gamma=\mathbb{Z}$. In this case, type $I-\mathbb{Z}-R N P$ is equivalent to type II-Z-RNP which, in turn, is equivalent to the Radon-Nikodym property. Type I-N-RNP is equivalent to type II-N-RNP and these properties are equivalent to the analytic Radon-Nikodym property [BD].

(iii) In general, it is unknown if type I- $M$-RNP and type II- $M-\mathrm{RNP}$ are equivalent properties for each subset $M$ of $\Gamma$. However, if $M$ is a Sidon set then this is true. Recall that $M$ is a Sidon subset of $\Gamma$ if $C_{M}(G)$ is isomorphic to $l^{1}(M)$, where $C_{M}(G)$ is the space of continuous functions on $G$ whose Fourier transform is supported on $M$, with the supremum norm. It is shown in [D1] that if $M$ is a Sidon subset of $\Gamma$ then a Banach space $X$ has type I- $M$-RNP if and only if it has type II- $M$-RNP if and only if it does not contain a copy of $c_{0}$.

The result that allows us to apply the ideas in $\S 2$ is the following result of Edgar [E]:

Proposition 3.1. A Banach space $X$ has type I-M-RNP if and only if for every bounded linear operator $T: L^{1}(G) / L_{M^{\prime}}^{1}(G) \rightarrow X$ the operator $T Q$ is representable, where $M^{\prime}=\{\gamma \in \Gamma: \bar{\gamma} \notin M\}$ and $Q: L^{1}(G) \rightarrow L^{1}(G) / L_{M^{\prime}}^{1}(G)$ is the natural quotient map.

Theorem 3.1. Let $1<p<\infty$, let $\left\{g_{n}\right\}_{n=1}^{\infty}$ be an approximate identity of positive functions in $L^{p}(G)$, and let $M$ be a subset of $\Gamma$ such that type I-M-RNP and type II-M-RNP are equivalent properties. Then the following statements are equivalent for a Banach space $X$ :

(a) $X$ has type I-M-RNP.

(b) For every operator $T \in \Lambda_{1}\left(L^{p}(G), X\right)$ with $T(\bar{\gamma})=0$ for all $\gamma \notin M$, the convolution $g_{n} * T$ converges to $T$ in $\Lambda_{1}\left(L^{p}(G), X\right)$.

Proof. Suppose $X$ has type I-M-RNP, and let $T \in \Lambda_{1}\left(L^{p}(G), X\right)$ with $T(\bar{\gamma})=$ 0 for all $\gamma \notin M$. Define a vector measure $\mu: \mathscr{B}(G) \longrightarrow X$ by

$$
\mu(E)=T\left(\chi_{E}\right) \quad \text { for all } E \in \mathscr{B}(G) .
$$

From this definition, it is easily seen that

$$
\int_{G} \varphi d \mu=T(\varphi) \quad \text { for all } \varphi \in C(G) .
$$

In particular,

$$
\hat{\mu}(\gamma)=\int_{G} \bar{\gamma} d \mu=T(\bar{\gamma})=0 \quad \text { for all } \gamma \notin M .
$$

By the same method of proof of Blasco's Theorem 2.1 [B2] we see that $\mu$ is of bounded variation and is absolutely continuous with respect to Haar measure 
$\lambda$. Now $X$ has type II- $M$-RNP, since type I- $M$-RNP and type II- $M$-RNP are equivalent, so there exists a function $f \in L^{1}(G, X)$ such that

$$
\mu(E)=\int_{E} f(t) d \lambda(t) \quad \text { for all } E \in \mathscr{B}(G)
$$

As noted by Blasco [B2], a standard argument shows that $f \in L^{q}(G, X)$, where $1 / p+1 / q=1$, and $T$ is represented by $f$ (see also [DU, p. 62]). Thus $g_{n} * T$ is represented by $g_{n} * f$. However, $g_{n} * f$ converges to $f$ in $L^{q}(G, X)$-norm so therefore $g_{n} * T$ converges to $T$ in $\Lambda_{1}\left(L^{p}(G), X\right)$ by the remark preceding Theorem 2.1.

Conversely, suppose (b) holds, and let $S: L^{1}(G) / L_{M^{\prime}}^{1}(G) \rightarrow X$ be a bounded linear operator. Define $T: L^{1}(G) \rightarrow X$ by $T=S Q$ where $Q: L^{1}(G) \longrightarrow$ $L^{1}(G) / L_{M^{\prime}}^{1}(G)$ is the natural quotient map. Then, clearly, $T(\bar{\gamma})=0$ for all $\gamma \notin M$. Again, the method of proof of Blasco's Theorem 2.1 [B2] shows that $T \in \Lambda_{1}\left(L^{p}(G), X\right)$. Thus, since (b) holds, $g_{n} * T$ converges to $T$ in $\Lambda_{1}\left(L^{p}(G), X\right)$. However, by Theorem 2.1(ii), $g_{n} * T$ is represented by functions $f_{n}$ in $L^{q}(G, X)$ and the sequence $\left\{f_{n}\right\}_{n=1}^{\infty}$ is a Cauchy sequence in $L^{q}(G, X)$ because $g_{n} * T$ converges to $T$ in $\Lambda_{1}\left(L^{p}(G), X\right)$. Therefore, $\left\{f_{n}\right\}_{n=1}^{\infty}$ converges in $L^{q}(G, X)$ to a function $f$ and $f$ represents the operator $T$. Hence $X$ has type I- $M$-RNP by Proposition 3.1.

Corollary 3.1. Let $1<p<\infty$ and let $\left\{g_{n}\right\}_{n=1}^{\infty}$ be an approximate identity of positive functions in $L^{p}(G)$. Then the following are equivalent for a Banach space $X$ :

(a) $X$ has the Radon-Nikodym property.

(b) For every operator $T \in \Lambda_{1}\left(L^{p}(G), X\right)$ the convolution $g_{n} * T$ converges to $T$ in $\Lambda_{1}\left(L^{p}(G), X\right)$.

Corollary 3.2. Let $1<p<\infty$, let $e_{n}(t)=e^{- \text {int }}$ for all $n \in \mathbb{Z}$, and let $\left\{g_{n}\right\}_{n=1}^{\infty}$ be an approximate identity of positive functions in $L^{p}(\mathbb{T})$. Then the following are equivalent for a Banach space $X$ :

(a) $X$ has the analytic Radon-Nikodym property.

(b) For every $T \in \Lambda_{1}\left(L^{p}(\mathbb{T}), X\right)$ with $T\left(e_{n}\right)=0$ for all $n<0$, the convolution $g_{n} * T$ converges to $T$ in $\Lambda_{1}\left(L^{p}(\mathbb{T}), X\right)$.

Corollary 3.3. Let $1<p<\infty$, let $M$ be a Sidon subset of $\Gamma$, and let $\left\{g_{n}\right\}_{n=1}^{\infty}$ be an approximate identity of positive functions in $L^{p}(G)$. Then the following are equivalent for a Banach space $X$ :

(a) $X$ does not contain a copy of $c_{0}$,

(b) For every $T \in \Lambda_{1}\left(L^{p}(G), X\right)$, with $T(\bar{\gamma})=0$ for all $\gamma \notin M$, the convolution $g_{n} * T$ converges to $T$ in $\Lambda_{1}\left(L^{p}(G), X\right)$.

Remarks. (i) Corollary 3.1 is the same as Blasco's Theorem 2.1 [B2] if $G=\mathbb{T}$.

(ii) Corollary 3.2 improves Blasco's Theorem 2.2 [B2] since it works for general approximate identities of positive functions in $L^{p}(\mathbb{T})$. 


\section{REFERENCES}

[B1] O. Blasco, Positive p-summing operators on $L^{p}$-spaces, Proc. Amer. Math. Soc. 100 (1987), 275-280.

[B2] - Convolution of operators and applications, Math. Z. 199 (1988), 109-114.

[BD] A. V. Bukhvalov and A. A. Danilevich, Boundary properties of analytic and harmonic functions with values in a Banach space, Mat. Z. 31 (1982), 203-214; English transl., Math. Notes Acad. Sci. USSR 31 (1982), 104-110.

[DU] J. Diestel and J. J. Uhl, Vector measures, Math. Surveys, no. 15, Amer. Math. Soc., Providence, RI, 1977.

[D1] P. N. Dowling, Duality in some vector-valued function spaces, Rocky Mountain J. Math. 22 (1992).

[D2] _ Radon-Nikodym properties associated with subsets of countable discrete abelian groups, Trans. Amer. Math. Soc. 327 (1991), 879-890.

[E] G. A. Edgar, Banach spaces with the analytic Radon-Nikodym property and compact abelian groups, Proceeding of the International Conference on Almost Everywhere Convergence in Probability and Ergodic Theory, Academic Press, Boston, MA, 1989, pp. 195-213.

[R] W. Rudin, Fourier analysis on groups, Tracts in Math., No. 12, Interscience, New York, 1962.

[S] H. H. Schaefer, Banach lattices of positive operators, Grundlehren Math. Wiss., vol. 215, Springer-Verlag, Berlin and New York, 1974.

Department of Mathematics and Statistics, Miami University, Oxford, Ohio 45056 